\title{
Volterra Kernel Interpolation for System Modeling and Predistortion Purposes
}

\author{
Peter Singerl ${ }^{*}$, Heinz Koeppl ${ }^{*}$ \\ * Christian Doppler Laboratory for Nonlinear Signal Processing \\ Graz University of Technology, Austria \\ ${ }^{\dagger}$ Infineon Technologies Austria AG \\ Email: \{peter.singerl, heinz.koeppl\}@tugraz.at
}

\begin{abstract}
RF power amplifiers of wireless communication systems are usually driven deep into their nonlinear region to obtain a higher efficiency. The price paid for the increased efficiency is the distortion of the transmission signal caused by the inhenent nonlinearity of the amplifier. To fulfill a given spectral mask for the transmission signa! and to avoid an unacceptable high bit-error rate, the amplifier must be linearized. One of the most powerful and efficient methods is djgital baseband predistortion. The predistorter must be operated on a sampling frequency which is sufficiently high to be able to compensate the outoff-band spectral components caused by the amplifier. Also for systemlẹvel simulations of communication systems, amplifier behavior models (high sampling rate) are used to estimate the genterated distortion. It is well known that nonlinear system identification can be accomplished on the Nyquist-rate regarding the input signal bandwidth, but however for several applientions, systems operating on the output signal Nyquist-rate are requiled. In this paper we show how such systems can be obtained by a multi-dimensional kernel interpolation without the drawbacks of a ligh condition number for the kernel estimation and the demand for an expensive high sampling rate ADC.
\end{abstract}

\section{INTRODUCTION}

To operate high power transmitters of RF communication systems with a higher efficiency, the final amplifier output-stages are usually driven deeply in their nonlinear region (approximate inverse relationship between power amplifier efficiency and linearity). The price paid for a higher efficiency is that the inherent nonlinearity of the RF power amplifier causes in-band distortion which degrades the bit error performance. It also causes spectral regrowth which leads to adjacent channel interference. Newer modulation formats such as WCDMA or OFDM are especially vulnerable to nonlinearities due to their high peak to average power ratio. In order to comply with spectral masks imposed by regulatory bodies and to reduce the bit enror rate. the $\mathrm{RF}$ power amplifier will be linearized. The most powerful and efficient linearization technique is digital baseband predistortion [1]-[3]. Fig. 1 depicts a simplified block diagram of a communication transmitter. The digital baseband predistorter distorts the input signal by a nonlinear operator which is ideally the inverse nonlinearity of the RF power amplifier to obtain an almost overall linear response up to the saturation point. To compensate the out-offband spectral components caused by the amplifier nonlinearity, the predistorter has to be operated on a sufficient high sampling frequency $\omega_{s}$ which is usually $N$-times higher than the transmission signal bandwidth. The factor $N$ denotes the highest order of the amplifier nonlinearity. Because the nonlinear behavior of the amplifier is in general unknown we apply a feedback path in Fig. 1 in order to identify either the amplifier and compute the inverse (predistorter) or to identify immediately the predistorter [4]-[6]. It is well known that the identification process can be accomplished on a sampling frequency which is just twice the input signal bandwidth [7]. However in both schemes, the predistorter is running on the high sampling frequency $\omega_{s}$ (see Fig. 1) to generate the out-off-band spectral

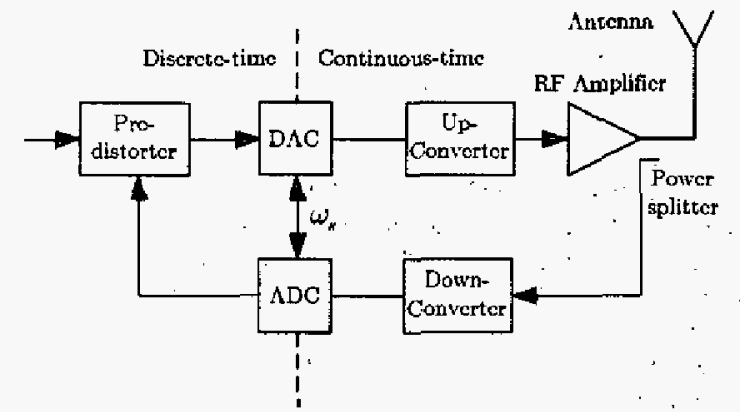

Fig. 1. Block diagram of a wireless comrnunication transmitter with digital baseband predistortion for the linearization of the RF power amplifier.

components required for the nonlinear compensation. The sampling frequency over twice the input signal bandwidth also plays a crucial role for the behavior modeling of physical RF power amplifiers. These models are usually used for efficient system-level simulations of the overall transmitter in the discrete-time baseband domain. To cover all out-off-band spectral components in the behavior model, the sampling frequency $\omega_{s}$ must also be $N$-times higher than the transmission signal bandwidth (without predistortion).

\section{[1. BASEBAND MODELING OF NONLINEAR PASSBAND SYSTEMS}

In system-level simulations, e.g. communication transmitters as depicted in Fig. 1, nonlinear behavioral models are often employed to predict the generated distortion (inter-modulation and spectral regrowth) caused by the inherent nonlinearities in physical devices without the full computational complexity of transistor based circuitlewel simulations [2]. Nonlinear passband systems like RF power amplifers can be modeled by a tandem connection of a dynamic nonlinear system and a linear passband filter as depicted in Fig. 2. The real passband signal $x(t)=|\tilde{x}(t)| \cos \left(\omega_{c} t+\phi_{0}(t)\right)$, where $\omega_{c}$ is the cartiet frequency, $\phi_{0}$ is the phase, and $|\bar{x}|$ is the amplitude, is fed to the nonlinear dynamic system described by the nonlinear system operator $\mathbf{H}$ to obtain the signal [8]

$$
\begin{aligned}
u(t) & =\mathbf{H}[x(t)]=\sum_{n=1}^{N} u_{+\varepsilon}(t) \\
u_{n}(t) & =\int_{0}^{\infty} \cdots \int_{0}^{\infty} h_{n}\left(\tau_{1}, \ldots, \tau_{n}\right) \prod_{i=1}^{n} x\left(t-\tau_{i}\right) d \tau_{i},
\end{aligned}
$$

where $h_{n}$ denotes the $n$ th-order time-domain Volterra kemel. The output signal is filtered by a linear 1st-zonal filter (real passband filter) [9], [10] described by the linear operator $\mathbf{F}$ to suppress the 
unwanted spectral components located around the multiples of the carrier frequency $\omega_{c}$ (see Fig. 3). Thetefore the output signal become

$$
y(t)=\mathbf{F H}[x(t)]=\sum_{n=1}^{N} \mathbf{F}\left[u_{n}(t) \mid,\right.
$$

which contains only the spectral components of interest near the carrier frequency $\omega_{c}$. If $\omega_{c}>B(2 N-1)$, where $2 B$ is the bandwidth of the bandpass signal $x(t)$, the complex baseband Volterra system [10], [11]

$$
\begin{gathered}
\bar{y}(t)=\sum_{k=0}^{[N / 2\rceil-1} \int_{0}^{\infty} \ldots \int_{0}^{\infty} \tilde{h}_{2 k+1}\left(\tau_{1}, \ldots, \tau_{2 k+1}\right) \\
\cdot \prod_{i=1}^{k+1} \tilde{x}\left(t-\tau_{i}\right) \prod_{i=k+2}^{2 k+1} \tilde{x}^{*}\left(t-\tau_{i}\right) d \tau_{1} \ldots d \tau_{2 k+1}
\end{gathered}
$$

exists. The signals $\bar{x}(t), \bar{y}(t)$ and the kemels $\bar{h}_{2 k+1}$ are the corresponding baseband and baseband-equivalent quantities [10]. The symbol * in (3) denotes complex conjugation. The baseband signals $\bar{x}(t)$ and $\bar{y}(t)$ are sampled with a sampling frequency $\omega_{o} \geq 2 N B$, which is at least $N$-times higher than the input-signal bandwidth to receive the full out-off-band spectrum without any aliasing (see Fig. 3). The output signal of the discrete-time nonlinear baseband model in Fig. 2

$$
\begin{gathered}
\hat{y}(n)=V \mid \bar{x}(n)]=\sum_{k=0}^{\lceil N / 2]-1} \sum_{l_{1}=0}^{L_{2 k+1}} \cdots \sum_{l_{2 k+1}=0}^{L_{2 k+1}} v_{2 k+1}\left(l_{1}, \ldots, l_{2 k+1}\right) \\
. \cdot \prod_{i=1}^{k+1} \tilde{x}\left(n-l_{i}\right) \prod_{i=k+2}^{2 k+1} \tilde{x}^{*}\left(n-l_{i}\right)
\end{gathered}
$$

is compared with $\tilde{y}(n)$ to create the error signal $e(n)$ which is minimized (MMSE) with respect to the discrete-time baseband Volterra kerneis $v$ in (4). The nonlinear model in (4) is the most general, which also includes the common simpler forms like Wiener, Hammerstein, Parallel Wiener and models which are described by purely static nonlinearities e.g. AM/AM- and AM/PM-conversion [2], [3], [12]. The components within the dashed-line box in Fig. 2 are usually not explicitly used e.g. in a system as depicted in Fig. 1 because the discrete-time baseband signals are provided by the baseband processor itself. With the structure depicted in Fig. 2 usually two major difficulties arises. The first is the demand for a high sampling rate Analog to Digital Converter (ADC) which is expensive and high power consuming. The second is the oversampling of the input signal $\dot{x}(n)$ by $N$, which leads to an non persistent excitation. This results in very large condition numbers and therefore to inaccurate Volterra kemel estimation if no retaliatory actions like regularization or truncated singular value decomposition (TSVD) are accomplished [13].

\section{BASEBAND MODELING OF NONLINEAR PASSBAND SYSTEMS BASED ON VOLTERRA KERNEL INTERPOLATION}

To overcome the difficulties mentioned in the last chapter, we identify the RF power amplifier on a sampling rate $\omega_{s} / N=2 B$ which satisfies the Nyquist theorem just for the band-limited $( \pm B)$ input signal $\tilde{x}(t)[7]$. Therefore the sampled output signal $\tilde{y}(n N)$ in Fig. 4 beceme aliased and will not anymore an equivalent representation of the continuous-time signal $\bar{y}(t)$. However we are able to identify the Volterra iremels of the nonlinear amplifier in Fig. 4 within the input-signal bandwidth in a unique way if the sampling rate satisfies the Nyquist theorem regarding the input signal $\tilde{x}(t)[7]$.

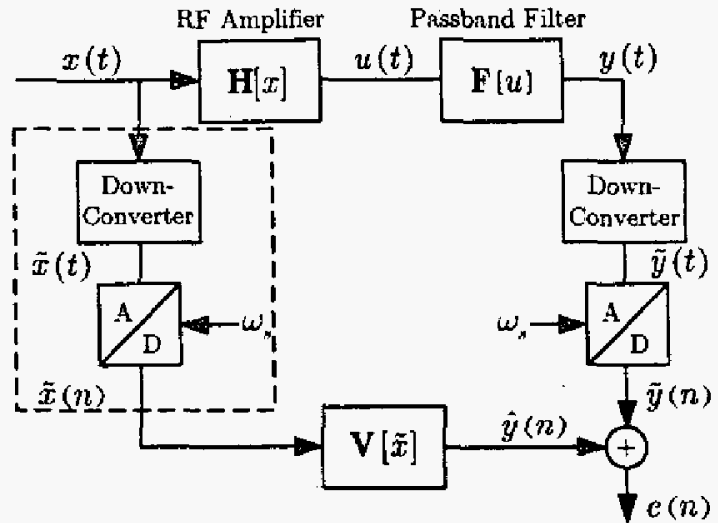

Fig. 2. Equivalent discrete-time baseband model generator $\mathbf{V} \mid \bar{x}\}$ for the continuous-time nonlinear passband system composed of $\mathbf{H}[x]$ and $F[u]$ for a sampling frequency $\omega_{s}$ which is at least twice the oupot signal bandwidth.

For this reason the output signal $\breve{y}(n)$ of the discrete-time model in Fig. 4 is in the ideal case (zero variance for the kernel estimation) identical to the sampled aliased output-signal $\vec{y}(n N)$. But for some applications e.g. digital predistortion or power amplifier modeling it is of fundamental importance to have a discrete-time system which is able to generate a non aliased output-signal as $\hat{y}(n)$ in Fig. 2 which contains the required out-off-band spectral components. Because it is not possible to calculate such a signal from the aliased signal $\breve{y}(n)$ in Fig. 4 we investigate the frequency-domain representation of the outpul-signal of the nonlinear discrete-time model $V$ in Fig. 2 in more detail. For this reason we consider the individual contributions to the time-domain signal $\hat{y}(n)$ in (4) for $k=0, \ldots,[N / 2\rceil-1$. If we span these signals in $2 k+1$ discrete-time variables we obtain

$$
\begin{aligned}
\hat{y}_{2 k+1}\left(n_{1}, \ldots, n_{2 k+1}\right)= & \sum_{l_{1}=0}^{L_{2 k+1}} \ldots \sum_{l_{2 k+1}=0}^{L_{2 k+1}} v_{2 k+1}\left(l_{1}, \ldots, l_{2 k+1}\right) \\
& \cdot \prod_{i=1}^{k+1} \bar{x}\left(n_{i}-l_{i}\right) \prod_{i=k+2}^{2 k+1} \tilde{x}^{*}\left(n_{i}-l_{i}\right) .
\end{aligned}
$$

To transform (5) to the frequency-domain, we apply a $(2 k+1)$ dimensional discrete Fourier transform to $(5)$, which yields the multidimensional frequency-domain signals

$$
\begin{aligned}
\hat{Y}_{2 k+1}\left(\omega_{1}, \ldots, \omega_{2 k+1}\right)= & V_{2 k+1}\left(\omega_{1}, \ldots, \omega_{2 k+1}\right) \\
& \cdot \prod_{i=1}^{k+1} \bar{X}\left(\omega_{i}\right) \prod_{i=k+2}^{2 k+1} \bar{X}^{*}\left(-\omega_{i}\right),
\end{aligned}
$$

which are periodic with $2 \pi$ in all frequency variables $\omega_{1}, \ldots, \omega_{2 k+1}$. The frequency-domain signals $\tilde{X}$ and $V$ are the Fourier transforms of the input signals and the time-domain Volterra kemels respectively. To calculate the $(2 k+1)$ th-order frequency-domain output signal. we apply an inverse Fourier transform to (6) which yields with some mathematical manipulations [14]

$$
\begin{aligned}
\hat{Y}_{2 k+1}(\omega)= & \frac{1}{(2 \pi)^{2 k}} \int_{-\pi}^{\pi} \cdots \int_{-\pi}^{\pi} \hat{Y}_{2 k+1}\left(\omega, \omega_{1}-\omega_{2},\right. \\
& \left., \omega_{2}-\omega_{3}, \ldots, \omega_{2 k}\right) d \omega_{1} \ldots d \omega_{2 k} .
\end{aligned}
$$

From (7) we recognize that the $(2 k+1)$ th-order frequency-domain output signal contribution of the Volterra system $\mathbf{V}$ in Fig. 2 is 


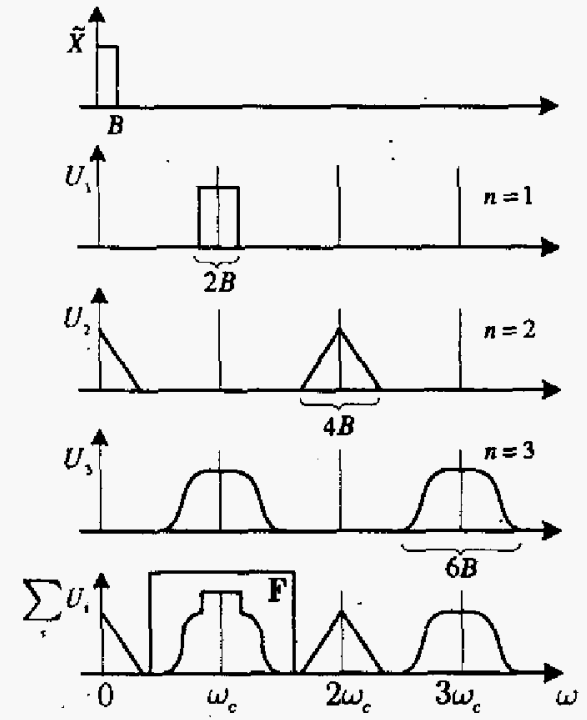

Fig. 3. Frequency-domain amplifiet output-signals with contributions from the different ordets of the amplifier nonlinearity up to $N=3$.

calculated by a kind of $2 k$-fold convolution of the band-limited multidimensional frequency-domain signal in (6). This process causes the desired spectral regrowth which is also present in the continuous-time amplifier output signal $\bar{y}(t)$. But it is important to note that we know from [7] that the system is uniquely defined by the Volterra kernels within the input signal bandwidth, because the kernels outside are masked by the band-linrited input signals $\bar{X}$ in (6). For this reason the frequency-domain Volterra kemels of the models in Fig. 2 and Fig. 4 satisfies $V_{2 k+1}\left(\omega_{1}, \cdots, \omega_{2 k+1}\right) \equiv \bar{V}_{2 k+1}\left(N \omega_{1}, \cdots, N \omega_{2 k+1}\right)$ for all $k$ 's within the input-signal bandwidth of $\pm \pi / N$. Therefore we can change the frequency-scaling of the volterra kemels $\bar{V}_{2 k+1}$ identified on the low rate in Fig. 4 with a factor of $N$ by a simple multidimensional zero-padding. The unwanted spectral copies caused by the zero-padding are masked (interpolation) by the band-limited high-rate input signal $\bar{X}(\omega)$. This process is depicted in Fig. 5 for a two-dimensional kemel for $N=2$, which was chosen for a convenient graphical representation although we do not have evenorder kernels in passband systems considered here. The shaded areas in Fig. 5 represents the non-zero values of the frequency-domain kemels. The multi-dimensional zero-padding is accomplished in the time domain by replacing all unit-sample delays in the discretetime Volterra system $\tilde{V}$ in Fig. 4 by $N$-sample delays. Therefore the new recalculated multi-dimensional Volterra kemels in Fig. 4 are expressed by

$$
\breve{V}_{2 k+1}\left(\dot{z}_{1}, \ldots, z_{2 k+1}\right)=\bar{V}_{2 k+1}\left(z_{1}^{N}, \ldots, z_{2 k+1}^{N}\right)
$$

where $z_{i}$ denoles the $Z$-domain frequency variables for $i=$ $1, \ldots, 2 k+1$. With the recalculated kemels in (8), we are able to construct a new Volterra model $\breve{\mathbf{V}}$ (see Fig. 4) which can be used in Fig. $2(\check{\mathbf{V}} \rightarrow \mathrm{V})$ to generate the discrete-time output-signal $\hat{y}(n)$ with the desired out-off-band spectral components. It is important that the recalculation of the low-rate identified kemels with the setup in Fig. 4 do not require any additional computational complexity. We only need some additional unit-sample delays (memory) which is in general not relevant because of its low implementation cost.

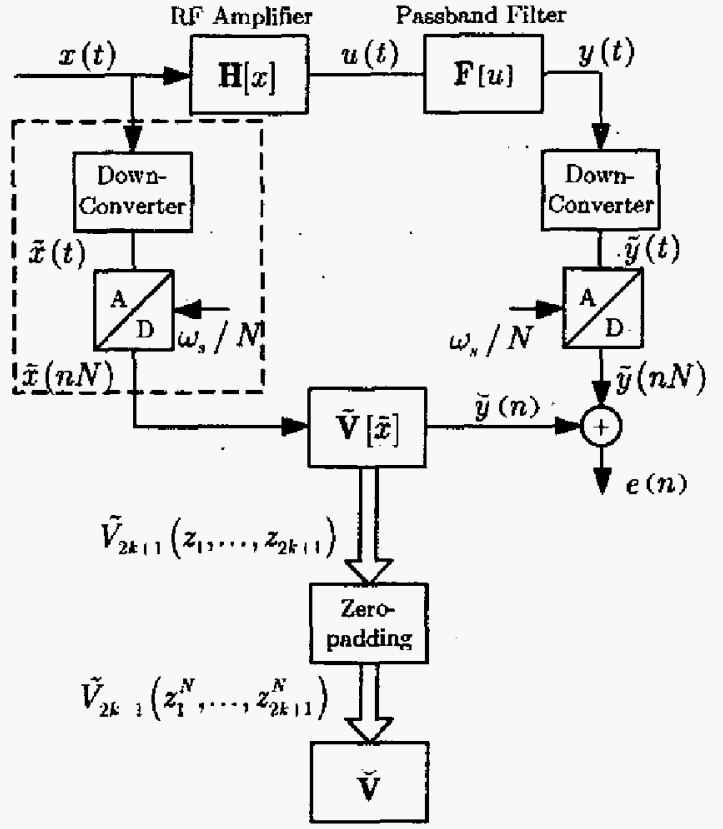

Fig. 4. Equivalent discrete-time baseband model generator $\breve{V}[\bar{x}(n)]$ for the continuous-time nonlinear passband system composed of $\mathbf{H}[x]$ and $F[u \mid$ for a sampling frequency $\frac{\omega_{A}}{N}$ which is at least twice the input signal bandwidth:

\section{SIMULATION RESULTS}

To validate the equivalence of the discrete-time nonlinear Volterra operators $\mathbf{V}[\tilde{x}(n)]$ and $\mathbf{V}[\bar{x}(n)]$ developed in the last chapter, we simulate the two different setups depicted in Fig. 2 and Fig. 4. For the RF amplifier we employ a device which generates negligible smatl highorder distortions ( $N=3$ ) to obtain illustrative frequency-domain result which are depicted in Fig. 6 . The amplifier will be excited with a band-limited ( $\pm 10 \mathrm{MHz}$ ) white Gaussian noise input sigral which generates an out-off-band distortion up to $30 \mathrm{MHz}$. The frequencydomain amplifier output signal from $\mathrm{V}[\tilde{x}(n)]$ resulting from the highrate identified kernels and the output signal $\breve{V}[\tilde{x}(n)]$ which results from the low-rate identified and interpolated (multi-dimensional zeropadding) kemels are depicted in Fig. 6 . These frequency-donain signals are almost identical as we have expected from the theoretical derivations shown in Sec. III (for a convenient representation only the magnitude is depicted here). The small difference which is caused by the nonzero variance (depends on the condition number depicted in Fig. 7) of the estimation process is depicted as a retative error in the lower part of Fig. 6. Because the model output signal in (4) is linear in the parameters, the unknown kernels can be estimated e.g. with standard linear-least-squares.

\section{CONCLUSION}

Although nonlinear systems can be identified on a sampling rate which is just twice the input signal band width, for some applications, nonlinear systems sampled on a rate which is twice the output signal bandwidth are necessary. One application is a digital predistorter which precedes the nonlinear amplifer to distort the amplifier input signal to obtain an almost overall linear response up to the saturation point. The digital predistorter must be able to generate out-off-band spectral components to compensate the spectral regrowth caused by the amplifier nonlinearity. Such high-rate models are also required for 


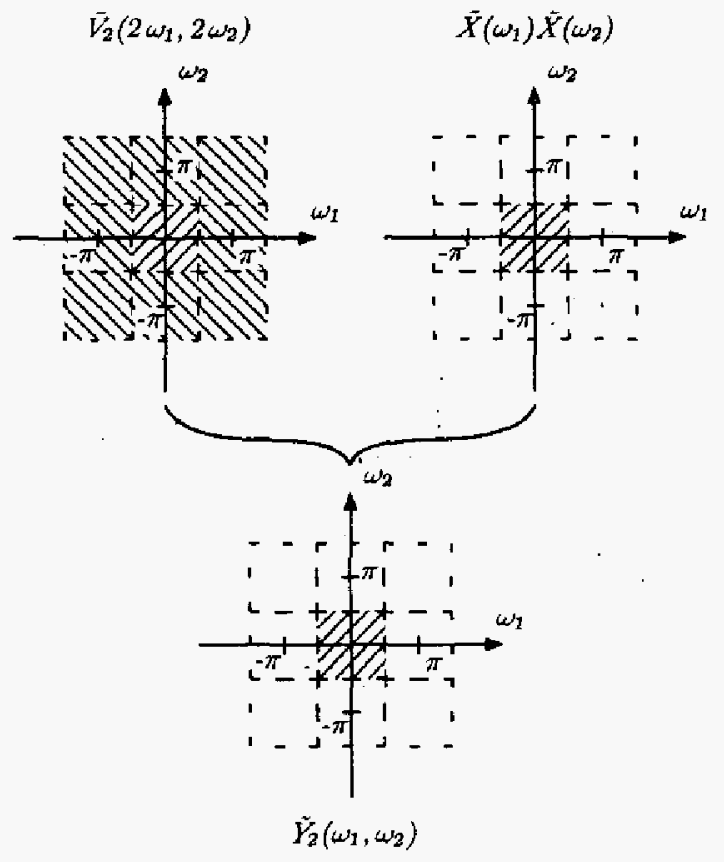

Fig. 5. Two-dimensional zero-padding and spectral masking by the highrate itwut-signal $\bar{X}\left(\omega_{1}\right) \bar{X}\left(\omega_{2}\right)$ for a two-dimensional Volterra kemel. The shaded areas illustrates the non-zero frequency-domain kemels
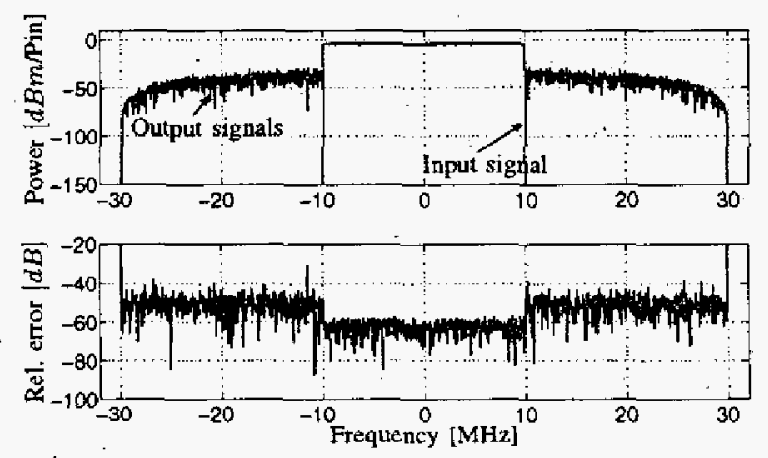

Fig. 6. Frequency-domain input and output signals from $\bar{x}(n), \mathbf{v}[\bar{x}(n)]$ and $\tilde{V}(\tilde{x})]$. The telative eror depicts the output signal difference caused by the volterra kemel estimation.

system-level simulations of a whole communication system to predict the generated distortion without the full computational complexity of a transistor-based circuit simulation. One method to construct such high-rate models is to identify a complex baseband system (predistorter, amplifier model) on a sampling frequency which fulfill the Nyquist theorem regarding the output signal bandwidth. One of the inherent problems with this method is the bad conditioned data matrix (large condition number) which leads to inaceurate kernel estimations (high variance). Another problem is the demand for a high sampling rate ADC which is expensive. To overcome these difficulties, we employ a novel method based on a multi-dimensional zero-padding of low-rate identified Volterra kemels. We have shown that this procedure requires no additional computational complexity. We only have to replace the unit-sample delays in the Votterra filter

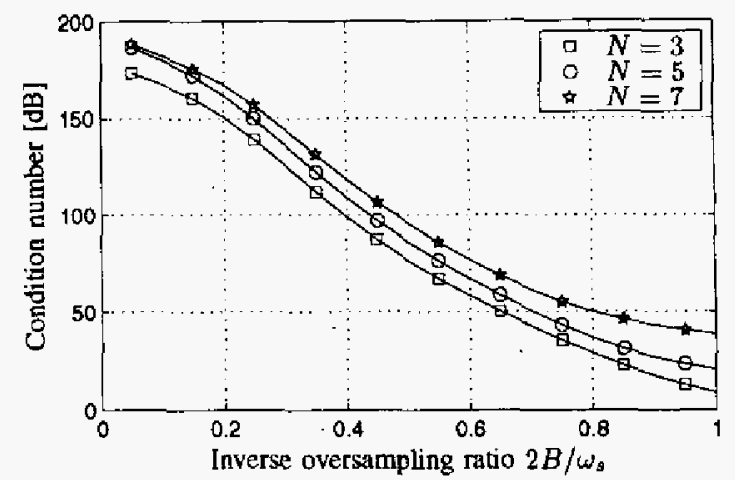

Fig. 7. The condition number, defined as the ratio of the biggest and the smailest singular value, determines the estimation accuracy. This number is small if the excitation, signal become white.

(predistorter, amplifier model) by $N$-sample delays. This increased demand for memory is usually not relevant because of its low implementation cost.

\section{REFERENCES}

[1] James K. Cavers, "Amplifier linearization using a digital predistorter with fast adaption and low memory requirennents," IEEE Trans. Veh. Technol, vol. 37, pp. 1885-1890, Dec. 1989.

[2] Peter B. Kennington, High linearity RF amplifier design, Artech House, Boston, London, 2000.

[3] Steve C. Cripps, RF power amplifiers for wireless communications, Artech House, Boston, London, 1999

[4] Raviv Raich, Hua Qian, and G. T. Zhou, "Digital baseband predistontion of nonlinear power amplifiers using orthogonal polynomials," in IEEE Intemational Conference on Acousfics, Speech, and Signal Processing, 2003. Pmoceedings. (ICASSP '03), Apr. 2003, pp. 689-692.

(5) Lei Ding, Raviv Rajch, and G. T. Zhou, "A Hammerstein predistortion linearization design based on the indirect learning architecture." in IEEE Intemational Conference on Acoustics, Speech, and Signal Processing. 2002. Proceedings. (ICASSP, 02), May 2002, pp. 2689-2692.

[6] Qian Yeqing. $\mathrm{Li} \mathrm{Qi}$, and Yao Tianren. "Analysis of different predistortion structures and efficient least-square adaptive algorithnss," IEEE Intemational Conference on Acoustics, Speech and Signal Processing. Apr. 2003, vol. 2, pp. 461-464.

[7] Walter A. Frank, "Sampling requirements for Volterta system identification," IEEE Signal Processing Letters, vol. 3. pp. 266-268. Sept. 1996.

[8] Wilsen J. Rugh, Nonlinear system theory. The johns hopkins university press, 2002.

[9] Nelson M. Blachman, "Detectors, bandpass nonlinearities, and their optimization: inversion of the Chebyshev transform" IEEE Trans. Inform. Theory, vol. 17, pp. 398-404, July 1971

[10] S. Benedetto, E. Biglieri, and R. Daffara, "Modeling and performance evaluation of nonlinear satellite links-a volterra series approach," IEEE Trans. Aerosp. Electron. Syst., vol. 15, pp. 494-507, July 1979.

[11] Raviv Raich and G. T. Zhou, "On the modeling of memory nonlinear effects of power amplifiers for communication applications," in Digiral Signal Processing Workshop and 2nd Signal Processing Education Workshop, Oct. 2002, pp. 7-10.

[12] V. John Mathews and Giovarni L. Sicuranza, Polynomial signal processing, John Wiley \& Sons, INC, 2000.

[13] Per Christian Hansen, Rank-deficient and discrete ill-posed problems. Numerical aspects of linear inversion. siam, Monographs on Mathematical Modeling and Computation, 1997.

[14] Martin Schetzen, The Volterra and Wiener theories of nonlinear systems, Krieger publishing company, Malabar, Florida, 1980. 